\title{
Les services médicaux d'aide à la reproduction peuvent-ils justifier leur discrimination envers les couples homosexuels?
}

\author{
Valérie VERNAEVE1, Patricia BAETENS², Oriol COLL1, Herman TOURNAYE2
}

1 Clinica Eugin, Barcelone, Espagne 2 Center For Reproductive Medicine, University Hospital, Dutch-speaking Brussels Free University (Vrije Universiteit Brussel), Belgique.

RÉSUMÉ

Bien qu'il existe de multiples moyens d'obtenir l'enfant désiré par les femmes homosexuelles, un nombre croissant de couples homosexuels se tournent vers les centres de médecine de la reproduction afin de réaliser des inséminations avec sperme de donneur. Cette pratique d'insémination de couples de femmes homosexuelles reste controversée dans le monde de la reproduction. Les données retrouvées dans la littérature ne démontrent aucun effet négatif sur le développement de l'enfant né au sein d'un couple homosexuel. Les seules objections à aider ces couples sont donc d'ordre purement éthique.

Mots clés : insémination par sperme de donneur, couples homosexuels

\section{INTRODUCTION}

Bien que l'insémination artificielle par sperme de donneur (IAD) soit l'une des techniques les plus anciennes et plus utilisées de la médecine de la reproduction, le débat sur qui peut avoir accès à cette technique continue. Initialement, cette technique a été utilisée dans le cas d'infertilité masculine ou en présence de maladie génétique auprès de l'homme. Depuis la montée des traitements alternatifs pour ce type de problème, comme l'ICSI et le diagnostic préimplantatoire, le nombre de couples hétérosexuels faisant appel à l'IAD a fortement diminué [4].

Depuis le début de la libération homosexuelle dans les années 60 , de plus en plus de femmes révèlent leur homosexualité et de ce fait ont attiré l'attention de la société [10, 14]. La majorité des mères lesbiennes concevaient leur enfant dans le contexte d'une relation hétérosexuelle. Souvent, les mères lesbiennes ne dévoilaient leur homosexualité qu'après la naissance de leurs enfants [5].

Depuis lors, de plus en plus de femmes lesbiennes ont leurs enfants dans le contexte d'une relation homosexuelle. Cette évolution a même été référée comme "Lesbian Baby Boom" [13].

Correspondance :

Dr. Valérie VERNAEVE - Clinica Eugin, Calle Entenza, 293-295, Bajos, 08029 Barcelone, Espagne Tel +34-93-322.11.22 - Fax +34-93-363.11.11 -

Email vvernaeve@euvitro.com 


\section{POSSIBILITÉS POUR LES COUPLES DE FEM- MES HOMOSEXUELLES DE VOIR SE RÉALISER LEUR DÉSIR D'ENFANTS}

\section{Relations sexuelles}

Les relations sexuelles, bien que souvent suggérées comme une manière "facile" pour obtenir une grossesse, est souvent une solution inacceptable pour les couples homosexuels. La majorité des couples considère cette option comme nuisible à leur relation car elle manque de respect pour l'identité du couple. De plus, certains problèmes liés à la reconnaissance de l'enfant peuvent surgir si l'homme soupçonne être le père de l'enfant porté par la femme. Il pourrait alors réclamer la paternité, et causer des problèmes au sein du couple homosexuel.

\section{L'adoption}

Dans les pays Européens, les couples homosexuels ne sont pas acceptés comme parents adoptifs sauf aux PaysBas. Dans des pays comme la Belgique et la France, le Code Civil ne considère que des couples hétérosexuels ou des femmes seules pour l'adoption.

\section{L'auto-insémination}

Certains couples homosexuels optent pour l'auto-insémination. L'auto-insémination n'implique pas l'interférence d'une équipe médicale et respecte dès lors plus l'autonomie et l'intimité du couple de lesbiennes. Une autre raison pour laquelle certains couples homosexuels privilégient l'autoinsémination est la préférence pour un donneur connu ou même pour un homme désirant assumer le rôle de père.

\section{L'insémination artificielle médicalisée}

Une autre possibilité permettant de devenir mère est l'IAD passant par l'aide d'une équipe médicale. Le dépistage médical des donneurs est une raison importante pour les couples homosexuels choisissant l'IAD, qui est considéré comme une procédure sûre. De plus, il n'est pas possible dans les pays Européens de légaliser la relation mèreenfant entre la mère non-biologique et l'enfant. L'utilisation d'un donneur anonyme est souvent justifiée par le désir du couple de lesbiennes de protéger la position du partenaire. Une autre raison importante est la protection de la relation de la partenaire en évitant l'interférence d'une troisième partie en utilisant du sperme anonyme $[1,7,11]$.

Néanmoins, le désir d'enfant des femmes homosexuelles suscite des controverses. C'est pourquoi la législation dans les pays Européens reflète les opinions différentes au niveau de qui peut avoir accès au traitement. En Autriche, en France, en Allemagne, en Norvège, en Suède et en Suisse, l'IAD est réservée aux couples hétérosexuels tandis que la législation en Israël, en Espagne et au Royaume-Uni autorise I'IAD pour les femmes célibataires. En général, la mère homosexuelle qui vit en couple sera toujours considérée comme une femme célibataire. Dans la majorité des pays européens sauf les Pays-Bas, le code civil exclut qu'un enfant ait deux parents du même sexe, donc la mère biologique aura toujours le statut juridique de mère céliba- taire. Juridiquement l'enfant ne pourra pas avoir de lien parental avec la mère non biologique.

La Belgique est un des pays européens où il n'y a pas de loi concernant les traitements de fertilité. C'est pourquoi les centres de fertilité en Belgique établissent leurs propres règles: qui est accepté pour un traitement, pour quelles raisons, et quel traitement est proposé, sont des décisions appartenant au centre même. Une proposition de loi à été introduite au sénat ce 2 mars dernier régularisant tous les procédés de la médecine de la reproduction en Belgique. Les centres Belges ne désirant pas aider ces couples homosexuels seront obligés de les diriger vers d'autres centres.

Dans le Centre de Médecine de la Reproduction de l'AZVUB, un comité éthique est installé pour évaluer chaque type de demande. La première demande d'une femme célibataire auprès du centre s'est présentée en 1981, suivie par la demande d'un couple de femmes lesbiennes. Le centre fut un des premiers à appliquer l'IAD à des couples homosexuels et des femmes célibataires. Le fondement de cette démarche résidait dans la conviction que chaque institution de santé doit étudier chacune des demandes de manière non discriminatoire et sans porter de préjugé. Le désir d'enfants peut être considéré comme un désir universel et légitime. Le fait d'être homosexuel ne peut exclure le désir d'être parent si ce désir tient compte du bien-être de l'enfant. Selon Robinson, les objections aux demandes des femmes célibataires ou homosexuelles sont plutôt des préjugés [15]. C'est pourquoi les objections ne justifient pas le refus à ces demandes.

Même en 1981, la parentalité homosexuelle n'était pas une chose nouvelle. Aux Etats-Unis le nombre de mères homosexuelles était estimé de 1 à 5 millions dont la majorité s'était retrouvée enceinte dans une relation hétérosexuelle précédente $[8,14]$. A cette époque les familles monoparentales étaient plus répandues et le désir d'enfant des femmes célibataires a suscité moins de critiques que celui des couples homosexuels. Néanmoins en 1981, le centre a encouru de nombreuses critiques pour sa position. Même, si dès le début, les femmes célibataires et les couples homosexuels ont été orientés vers un psychologue afin d'évaluer chaque demande. En même temps il nous paraissait souhaitable de suivre le développement des enfants dans ces familles alternatives.

\section{LE SUIVI DES ENFANTS DE COUPLES HOMOSEXUELS}

En ce qui concerne les enfants de couples de femmes lesbiennes, la recherche empirique ne confirme pas les suppositions théoriques. Les premières recherches datent des années quatre-vingt. Ces recherches ont été faites surtout aux Etats-Unis dans le cadre des procédures de divorce des femmes homosexuelles, parce qu'elles risquaient de perdre le droit de garde de leurs enfants. Ceci implique que ces enfants ont passé quelque temps dans une famille hétérosexuelle et qu'ils ont vécu le divorce de leurs parents. Souvent, les enfants étaient restés en contact avec leurs 
pères. Ces recherches n'ont donc pas porté beaucoup d'attention sur le rôle de la compagne de la mère homosexuelle. Néanmoins, ces recherches nous prouvaient qu'accepter les couples homosexuels pour l'IAD ne s'opposait pas nécessairement au bien-être des enfants dans ce type de famille $[2,5]$.

Récemment de plus en plus des recherches sur les enfants qui sont nés dans le contexte d'une relation lesbienne ont été menées. Ces enfants grandissent dès leur naissance dans des familles où deux femmes partagent la responsabilité de l'éducation de l'enfant. Ces enfants n'ont pas vécu le divorce de leurs parents. Par contre la majorité de ces enfants ont été conçus avec le sperme d'un donneur anonyme et ils n'ont jamais eu de père. Ces recherches sont relativement unanimes dans leurs résultats.

La satisfaction relationnelle, la durée de la relation et le nombre de séparations sont les mêmes pour les couples homosexuels comparés aux couples hétérosexuels $[4,8]$. Généralement les mères homosexuelles avaient une meilleure interaction avec leurs enfants que les parents hétérosexuels $[4,8,9,18]$. Les recherches qui ont comparé le rôle de la compagne de la mère à celui du père dans les familles hétérosexuelles ont trouvé que les mères nonbiologiques étaient plus impliquées dans les activités éducationnelles y compris les actions disciplinaires $[4,8,18]$. Le développement socio-psychologique des enfants nés dans des familles lesbiennes était similaire à celui des enfants des familles hétérosexuelles. II n'y avait pas de différences au niveau de leur développement psychosexuel $[4,13]$. En plus il n'y avait pas plus de problèmes émotionnels, ni de problèmes comportementaux $[4,6,8,9,12,13$, 16].

Une étude récente à comparé 37 enfants de 10 ans et 5 mois issus d'un couple homosexuel à 37 enfants du même âge issu d'un couple hétérosexuel. Cette étude nous montre que le même nombre d'enfants dans les deux groupes ont été sujets à des moqueries. Cette étude démontre également que l'estime de soi et le bien être général des enfants issus de couples homosexuels est identique aux enfants issus de couples hétérosexuels [17].

Les résultats de ces études prouvent que les enfants issus de couples homosexuels ne courent pas nécessairement plus de risques que les enfants des couples hétérosexuels. En plus ces recherches prouvent qu'en général les deux partenaires partagent la responsabilité de l'éducation de l'enfant. La mère homosexuelle, si elle vit en couple, ne fait donc pas d'enfant toute seule malgré le fait que juridiquement elle ait le statut de mère célibataire.

\section{LES DEMANDES D'IAD PAR DES COUPLES DE FEMMES HOMOSEXUELLES}

En 2004, 420 nouveaux couples ont débuté un traitement par IAD au sein du Centre de Reproduction Humaine de l'AZ-VUB. De ces couples, 225 sont des couples homosexuels $(53,6 \%)$. De ces 225 couples homosexuels, 123 nous venaient de France $(54,7 \%)$ et 102 de Belgique
$(45,3 \%)$. L'Espagne est également un pays vers lequel beaucoup de couples de lesbiennes Française se dirigent. A la Clínica Eugin, 73 nouveaux couples homosexuels se sont présenté en 2004 en vue d'une IAD. Soixante-deux couples étaient originaires d'autres pays que de l'Espagne (85\%). Cinquante-cinq couples étaient en provenance de France $(75 \%)$.

Une étude comprenant le suivi de 95 couples homosexuels belges ayant fait une demande d'IAD entre 1992 et 1999 a été réalisée à l'AZ-VUB [3]. Six couples ont été refusés pour le traitement et 12 couples ne sont pas revenus pour le traitement. La durée de la relation était en moyenne de 5,45 ans avec un écart-type de 3,55 et tous les couples vivaient ensemble. Au moment de la demande $76,8 \%$ des mères biologiques et $89,5 \%$ des partenaires se considéraient exclusivement homosexuelles. Les couples étaient acceptés par $83,2 \%$ des parents des mères biologiques et $81,7 \%$ des parents de la mère sociale. $92,6 \%$ des couples vivaient dans un réseau social plutôt hétérosexuel. Seulement $15,8 \%$ des couples étaient membres d'une association d'homosexuels.

Le désir d'enfants existait depuis plusieurs années chez $81,1 \%$ de ces couples. Chez les mères biologiques, le désir d'enfants existait déjà de manière significative en dehors de la relation actuelle tandis que pour leurs partenaires ce désir était lié à la relation actuelle $(u=3414,5 ; p<0.005)$ mais toutes les mères biologiques avaient attendu le partenaire avec qui elles pouvaient réaliser ce désir d'enfant. $55,8 \%$ des couples étaient dès le début, convaincus que I'IAD était la meilleure solution. Les autres couples avaient pensé à d'autres solutions comme un donneur direct. Le choix pour l'IAD était motivé par le fait qu'on ne voulait pas introduire une tierce personne dans la famille et par peur qu'un donneur direct pourrait exiger des droits paternels. Tous les couples avaient un désir d'enfant commun mais seulement chez $13,7 \%$ des couples les deux partenaires avaient le désir de grossesse. Chez $74,7 \%$ des couples seulement une des partenaires désirait être enceinte, mais cette situation est typique chez les couples belges. Une situation qui est aussi typique surtout pour les couples flamands était que $54,7 \%$ des familles homosexuelles étaient considérées comme une famille de deux mères et que $51,6 \%$ des couples apprendrait à leurs enfants à les appeler par deux noms synonymes du mot 'maman'. Les autres couples $(43,2 \%)$ considéraient que leur famille se composait d'une mère et d'un partenaire qui aurait un rôle parental mais différent de celui de la mère. Mais tous les couples étaient d'avis de partager la responsabilité de l'éducation de leurs enfants d'une manière égalitaire et équilibrée.

La majorité des couples homosexuels étaient donc des couples stables et le désir d'enfant était un désir commun des deux partenaires.

\section{PRISE EN CHARGE PRATIQUE DES COUPLES HOMOSEXUELS AU CENTRE DE REPRODUCTION HUMAINE DE L'AZ-VUB}

Vu les résultats rassurants du devenir des enfants nés au 
sein de couples homosexuels, l'évaluation des couples par la psychologue a été abandonnée. Actuellement, l'évaluation des couples se fait par une infirmière préalablement formée en utilisant un questionnaire. Ce questionnaire a pour but d'évaluer la situation familiale des deux partenaires, leur orientation sexuelle antérieure, la relation avec les membres de leur famille réciproque, la relation avec le cercle d'amis et voisinage, leur situation professionnelle. Sont également évaluées les motivations de parentalité, la future organisation de la parentalité et l'attitude envers l'absence de père.

Au sein de la Clinique EUGIN, le protocole suivi par les femmes homosexuelles est identique à celui des demandeuses d'insémination artificielle avec sperme du conjoint ou de donneur.

Le traitement proprement dit se fait à l'aide d'une superovulation avec du citrate de clomifène ou régime à faible dose de gonadotrophines avec un contrôle du cycle à l'aide d'échographies et bilans hormonaux. Un taux de grossesse cumulé de $60 \%$ peut être espéré après trois tentatives (Figure 1).

\section{CONCLUSION}

Nous pouvons donc conclure qu'il n'y a pas d'inconvénient scientifique contre I'IAD auprès de couples de femmes homosexuelles et que les taux de réussite élevés de cette technique permet d'aider beaucoup de couples à avoir l'enfant désiré en limitant les risques médicaux et psychologiques que d'autres solutions pourraient engendrer.

\section{REFERENCES}

1. BAESTEN P., PONJAERT-KRISTOFFERSEN I., VAN STEIRTEGHEM A.C., DEVROEY P. : Counselling lesbian couples in creating a family by artificial insemination by donor. Hum. Reprod., 1996, 11 (abstract) : 79.

2. BAESTEN P., BREWAEYS, A. : Lesbian couples requesting DI. An update of the knowledge with regard to lesbian mother families. Hum. Reprod. Update, 2001, 7:512-519.

3. BAESTEN P., CAMUS M., DEVROEY P. : Counselling lesbian couples : requests for donor insemination on social grounds. R.B.M. Online, 2002, $6: 75-83$.

4. BREWAEYS A., PONJAERT I., VAN HALL E., GOLOMBOK S.: Donor insemination : child and family development in lesbianmother families with children of 4- to 8 years old. Hum. Reprod., 1997, 12 : 1349-1359.

5. BREWAEYS A., VAN HALL E.V. : Lesbian motherhood : the impact on child development and family functioning. J. Psychosom. Obstet. Gynaecol., 1997, 18 : 1-16.

6. CHAN R., RABOY B., PATTERSON C.J. : Psychosocial adjustment among children conceived via donor insemination by lesbian and heterosexual mothers. Child Dev., 1998, 69 : 443457.

7. ENGLERT Y. : Artificial insemination of single and lesbian women with donor semen. Hum. Reprod., 1994, 9 : 1969-1971.

8. FLAKS D., FICHER I., MASTERPASQUA F., JOSEPH, G. Lesbians choosing motherhood : a comparative study of lesbian and heterosexual parents and their children. Dev. Psychol., 1995, 31 : 105-114.

9. GOLOMBOK S., TASKER F., MURRAY C. : Children raised in fatherless families from infancy: family relationships and the socioemotional development of children of lesbian and single heterosexual mothers. J. Child Psychol. Psychiatry, 1997, 38 : 783-791.

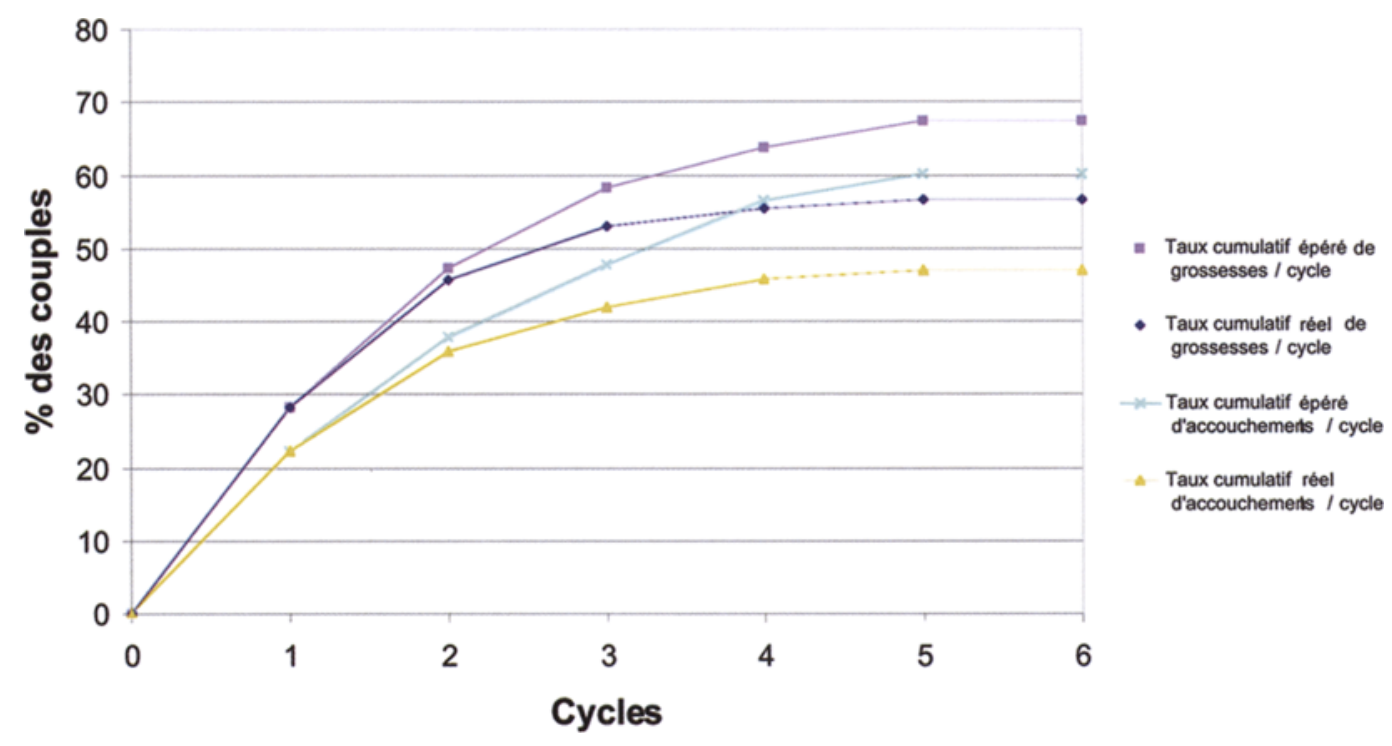

Figure 1 : Résultats des inséminations intra-utérines par sperme de donneur auprès de couples homosexuels.

Taux cumulatifs, espérés et réels, de grossesses et d'accouchements dans des cycles d'insémination avec stimulation par citrate de clomifène et hCG (81 couples ayant entamé le traitement). 
10. HITCHENS D. : Social attitudes, legal standards and personal trauma in child custody cases. J. Homosexuality, 1980, $5: 89$ 95.

11. JACOB M.C. : Lesbian couples and single women. In : Burns L.H., Covington S.H. eds. Infertility Counselling. A comprehensive Handbook for Clinicians. New York, Parthenon, 1999.

12. MCCANDLISH B. : Against all odd: lesbian mother dynamics. In: Bozett F. ed. Gay and lesbian parents. New York, Praeger, 1987.

13. PATTERSON C.J. : Children of the lesbian baby boom: behavioural adjustment, self concept and sex role identity. In : Greene H., Herek G.M. eds. Lesbian and Gay Psychology, Theory, Research and Clinical Applications. Newbury Park, Sage, 1994.

14. PATTERSON C.J. : Children of gay and lesbian parents. In : Ollendick T.H. and Prinz R.J. eds. Advances in Clinical Child Psychology. New York, Plenum Press, 1997.

15. ROBINSON B. : Birds do it. Bees do it. So why not single women and lesbians ? Bioethics, 1997, $11: 217-227$.

16. STECKEL A. : Psychosocial development of children of lesbian mothers. In : Bozett F. W. ed. Gay and lesbian parents. New York, Praeger, 1987.

17. VANFRAUSSEN K. : What does it mean for youngsters to grow up in a lesbian family created by means of donor insemination? J. Reprod. Infant Psychol., 2002, 20 : 237-254.

18. VANFRAUSSEN K., PONJAERT-KRISTOFFERSEN I., BREWAEYS A. : Family functioning in lesbian families created by donor insemination. Am. J. Orthopsychiatry, 2003, $73: 78-90$.

Communication au XII/ Séminaire de la Fédération Française des CECOS, Tours, 7-9 Avril 2005.

Manuscrit reçu : juin 2005 ; accepté juillet 2005.

\section{ABSTRACT}

Can fertility doctors justify their intolerance

of homosexual couples? Valérie VERNAEVE, Patricia BAETENS, Oriol COLL,
Herman TOURNAYE,

Although lesbian women have a variety of ways to become mothers, an increasing number of lesbian couples attend fertility centres asking for donor insemination. The practice of inseminating lesbian couples remains a controversial topic within the reproductive medicine world. A review of the literature did not reveal any harmful effects of lesbian motherhood on child development. Furthermore, this treatment is highly effective with an expected cumulative pregnancy rate of $60 \%$ after three intra-uterine inseminations with superovulation using clomifene citrate. The only objection to medically assisted procreation in these couples is therefore purely ethical. The extensive experience of the AZ-VUB Centre for Reproductive Medicine that played a pioneer role in this field, is illustrated in this article.

Key words: donor insemination, lesbian couples 